\title{
Semantic Segmentation Based Remote Sensing Data Fusion on Crops Detection
}

\author{
Jose Pena',2, Yumin Tan'1, Wuttichai Boonpook ${ }^{1}$ \\ ${ }^{1}$ School of Transportation Science and Engineering, Beihang University, Beijing, China \\ ${ }^{2}$ Venezuela Space Agency (ABAE), Caracas, Venezuela \\ Email: josepenaoliveros@yahoo.com
}

How to cite this paper: Pena, J., Tan, Y.M. and Boonpook, W. (2019) Semantic Segmentation Based Remote Sensing Data Fusion on Crops Detection. Journal of Computer and Communications, 7, 53-64. https://doi.org/10.4236/jcc.2019.77006

Received: May 20, 2019

Accepted: July 7, 2019

Published: July 10, 2019

\begin{abstract}
Data fusion is usually an important process in multi-sensor remotely sensed imagery integration environments with the aim of enriching features lacking in the sensors involved in the fusion process. This technique has attracted much interest in many researches especially in the field of agriculture. On the other hand, deep learning (DL) based semantic segmentation shows high performance in remote sensing classification, and it requires large datasets in a supervised learning way. In the paper, a method of fusing multi-source remote sensing images with convolution neural networks (CNN) for semantic segmentation is proposed and applied to identify crops. Venezuelan Remote Sensing Satellite-2 (VRSS-2) and the high-resolution of Google Earth (GE) imageries have been used and more than 1000 sample sets have been collected for supervised learning process. The experiment results show that the crops extraction with an average overall accuracy more than $93 \%$ has been obtained, which demonstrates that data fusion combined with DL is highly feasible to crops extraction from satellite images and GE imagery, and it shows that deep learning techniques can serve as an invaluable tools for larger remote sensing data fusion frameworks, specifically for the applications in precision farming.
\end{abstract}

\section{Keywords}

Data Fusion, Crops Detection, Semantic Segmentation, VRSS-2

\section{Introduction}

At present RS technology has received great attention in the agriculture community due to its ability to provide periodic and regional information for crop monitoring and thematic mapping [1] [2]. Modern RS to identify any features 
on the surface is no longer considered as a processing of a one-source single date image. It has shifted to multi-source fusion of multi-temporal images. Several spectral indices have been proven to be valuable tools in describing crop spatial variability. In this context, the images of high spatial and spectral resolution have already proved their potential and effectiveness in crop detection. However, when we are considering to identify the types of crops with multispectral imageries, RS becomes more challenging. The main challenge of satellite based remote sensing application in agricultural field at present is that there is no suitable sensors with very high spatial resolution like below 50 centimeters and with a good temporal resolution and spectral resolution at the same time.

Indeed, novel approaches and algorithms using Unmanned Aerial Vehicle (UAV) or satellite based multispectral imaging have been developed for vegetation classification [3]. But, the acquisition of UAV images or images of other platform such as GeoEye-1, WorldView-4 and KompSat 3a can be difficult to acquire considering the high cost, and their availability only in the specific small region. Google Earth (GE) provides an open data source with very high spatial resolution, which represents a very good alternative for crops detection. Very few studies have been undertaken to use GE images as the direct data source for land use/cover mapping [4]. Numerous methods based on DL have been proposed recently for agricultural applications over specific RS data, especially focusing on high resolution and hyperspectral images [5], plant phenotyping [6] or weed scouting [7] and early disease detection [8]. However, some recent approaches have tried to directly adopt deep architectures designed to identify other aspects related to the vegetation or the diseased plants, the results, although very encouraging, appeared coarse [9].

In this research, we are going to identify several types of crops that has very different shapes, sizes, and color intensities, and the surrounding plants and background soil strongly differ across regions. In addition, data fusion of RGB images (with high spatial resolution) obtained from Google platforms and multispectral satellite imagery obtained from Venezuelan Remote Sensing Satellite (VRSS-2), will be done through Gram-Schmidt (GS) pan-sharpening method. Fusion images and vegetation indices (VIs) were used as input to following SegNet based semantic segmentation. Our main contributions can be summarized as: this is probably the first attempt conducted to explore the combination of VRSS-2 and GE imagery, through a data fusion process for crop detection; a SegNet-based semantic segmentation model is proposed for crop type detection, capable of adapting to fusing data sets in which the results proved that this approach provide better performance than that of the traditional classification methods; a self-designed preparation of data sets and semantic segmentation network have been employed to provide a per pixel labelling of the input data; finally, two different data sets from VRSS-2 and GE, those are obtained absolutely free of cost, have been employed with several pre-processing and post-processing strategies, designed and combined with Segnet architecture, that has increased the overall accuracy. 


\section{Materials and Methods}

\subsection{Study Area}

The study area is located in the north-central region of Venezuela, Aragua State, Palo Negro Sector-Venezuela. The most important agricultural production is concentrated in this area and the main crops produced are banana, pasture, papaya and coco. Banana and pasture production have greater importance in the study area, because they represent $65 \%$ of the economy of that region of Venezuela. In recent years their production and thereby, the source of employment have been declined considerably. Reasonably, the state has taken steps to identify and quantify the possible reasons and overcome the problems. 'Bare land' comes into this issue as one of the solutions to increase their production using that lands which are in plenty. In this study, different training zones and testing zone are used.

\subsection{Data Sets Construction}

The design of the training dataset is the key to the performance of a good CNN classification model, and the construction of dataset is described below. All three datasets used in this research are contained the RGB image set from VRSS-2 image, Google earth mapping, and data fusion images which are composed of the multispectral bands including RGB bands, Near-infrared (NIR), and normalized difference vegetation index (NDVI).

\subsubsection{VRSS-2 Image}

VRSS-2 was launched on October 09, 2017, and owned by the Bolivarian Agency for Space Activities (ABAE). It contains two different cameras, High Resolution Camera (panchromatic and multispectral sensors) and Infrared Camera. VRSS-2 data has a total of 10 bands including a panchromatic band (band 1) which has 1 meter of spatial resolution, nine multispectral bands (band $2-10$ ) which has the spatial resolution in 3 meter (band 2 - 5), $30 \mathrm{~m}$ (band 6 - 8) and 60m (band 9 10) respectively. However, in this research, only five bands are selected (bands 1 - 5).

The radiometric calibration procedure is first applied to selected VRSS-2 images to generate a consistent output images. To obtain the high-quality fusion data, it is important to apply the data corrections for various lighting conditions such as overcast skies and partial cloud coverage. To correct for this aspect, we utilize sunlight sensors measuring the sun's orientation and sun irradiance, as shown in Figure 1.

The obtained data are stored as quantized and calibrated Digital Numbers (DN). The DN are converted to surface reflectance value using the Equation (1) with coefficients provided in metadata file and ABAE.

$$
\rho^{T O A}=\pi \times L \times d^{2} /\left(E_{\text {sum }} \times \cos \left(\theta_{s}\right)\right)
$$

where, $\rho^{T O A}$ is surface reflectance of the earth at the top of the atmosphere, $L$ is apparent radiance at the top of the atmosphere in Watt $/ \mathrm{m}^{2} /$ stereo-radian/ 


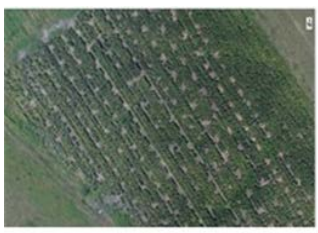

(a)

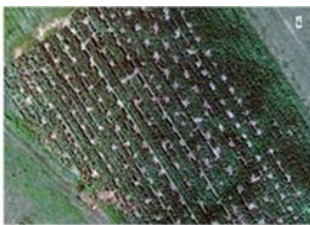

(b)

Figure 1. Radiometric calibration pattern samples. (a) Image without calibration and (b) image after calibration.

micrometers, $d^{2}$ is average distance between the earth and the sun in atmospheric units (UT), $E_{\text {sum }}$ is ex-atmospheric irradiation in watt $/ \mathrm{m}^{2} /$ micrometers and $\cos \left(\theta_{s}\right)$ is cosine of the angle of the solar elevation. Once the Top of Atmosphere (TOA) reflectance of required bands are calculated, they are stacked to get one single image.

\subsubsection{Google Earth Imagery}

Google Earth provides open and highly spatially resolved images. This platform contains petabyte-scale data for scientific analysis and visualization, easily accessible and user-friendly, and a convenient environment for interactive data or algorithm development. However, GE images have rarely been used as the main data source to identify crops. GE images are limited to a three-band color code ( $R, G, B)$, which is expected to lower the classification performance due to its poor spectral signature [10]. Actually, the potential for the classification of spatial characteristics by Google Maps has been underestimated [11].

\subsubsection{Data Fusion}

The purpose of data fusion is to obtain more accurate and more abundant information than any single dataset by processing multi-source redundant data in space and time according to certain algorithms, and then generate combination images with new space, spectrum, and time characteristics [12]. In this study, the first implementation of data fusion is done between band 1 and band $2-5$ of VRSS-2 which were captured under 0\% cloud cover on 16 December 2018. The multispectral bands have a native spatial resolution of $3 \mathrm{~m}$, but they are pan sharpened to $1 \mathrm{~m}$ by using the panchromatic band. The Gram Schmidt (GS) pan-sharpen method was used in this process. In the second implementation of data fusion, the Google earth images which have the high resolution up to $50 \mathrm{~cm}$ were fused with the infrared band of above fused VRSS-2 images. The result of data fusion is multispectral bands (R, G, B, NIR). After the two fusions, crop recognition accuracy could be increased to some degree, but it is still hard to differentiate similar crops by traditional classification. Therefore, to improve the crop detection precision, Normalized Difference Vegetation Index (NDVI) is computed and used, which could enhance the spectral information and increasing the separability of the interested classes.

\subsection{Methods}

This Section describes the proposed methodology for crop detection. It is di- 
vided into three steps: First, fusion between two selected remote sensing imageries are made and NDVI band is computed, and then these newly produced images are used as input in the training dataset labeling. Second, we have employed a self-designed data sets and semantic segmentation network to provide a per pixel semantic labeling of the input data. Finally, the accuracy assessment is conducted. Two experiments are designed and implemented in this research according to the different datasets used.

\subsubsection{Data Preparation}

SegNet architecture cannot directly deal with one scene of VRSS-2 image, due to the limitation of GPU memory and other factors. Resizing images to a proper size is a common idea, but it will result in the loss of large amounts of information. In this paper, we propose a patch-wise to deal with this problem. Firstly, the VRSS-2 image was resized into 120 scenes of $3200 \times 2400$ pixels which are at the same amount and size as the Google scenes, which are subsequently merged. Secondly the image was split into many local patches with an appropriate size according to the dataset and the GPU capacity (the size of a local patch for our dataset was 480 x 480 pixels). Sample datasets can be seen in Figure 2.

As to the dataset production, we first separated all images used for training into three semantic classes and labeled them as banana, pasture and bare land separately. The challenges in creating such datasets lie in that there are so many patterns, different shapes and colors, confusing details caused by low attitude, and complex land cover types in the object area. The sample dataset is show in Figure 3. In our experiment, we divide these annotated datasets into three sets:

(a)

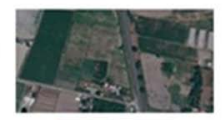

(b)

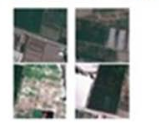

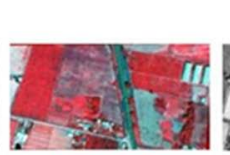

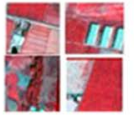

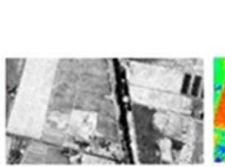

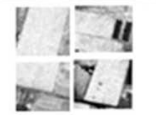

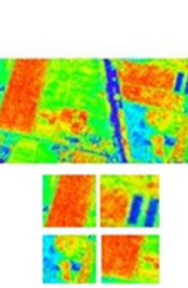

3200

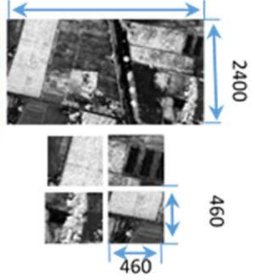

Figure 2. Samples with different size, band combination. (a) Image bands with $3200 \mathrm{x}$ 2400 pixels and from left to right: true color, color-infrared, NDVI, GNDVI and SPEAR Vegetation Delineation, (b) according bands with $480 \times 480$ pixels.

(a)

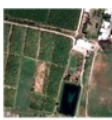

(b)

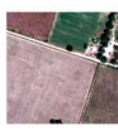

(c)

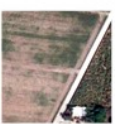

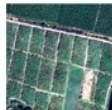
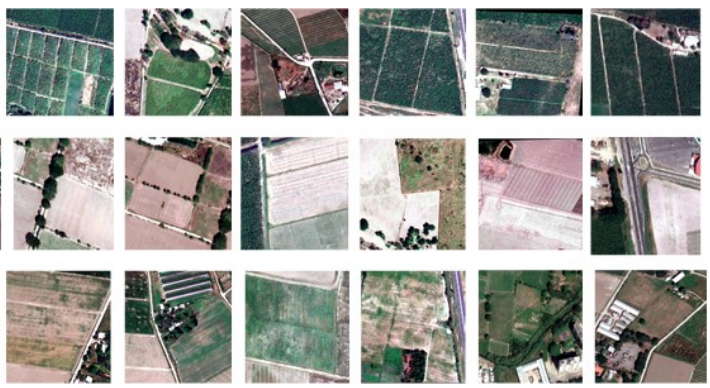

Figure 3. Data fusion samples, where different crops areas are visualized. (a) Banana crops, (b) bare land area and (c) pasture zones. 
750 sets for training, $20 \%$ of randomly selected from the training set for validating, and 50 sets for testing. The testing set is captured from different areas compared to the training sample set.

Figure 3(a) shows some scenes with banana plantation where you can see different levels of growth, even within the plantations there are irregular areas without crops. Figure 3(b) shows the bare lands with various color and some sample observations of different types of land conversions. The Figure 3(c) shows diverse kinds of pasture with mixed colors, managed with agricultural practices of seeding, irrigation and with practices like controlled burning and regulated intensity of grazing. In order to annotate the crop types, Labelme software is selected in this research to label and design a preprocessor for building labeled dataset. By artificial labeling, a large number of annotations for different crops classes was created. All data are classified into four datasets. The first dataset bases on the data fusion of VRSS-2 satellite images between pan-sharpening image with multispectral bands (R, G, B), which is called VRSS-2 dataset here. The second dataset as named GI is the RGB image from Google earth imagery which consists of RGB bands. The third dataset is the data fusion of VRSS-2 with Google earth images, which is called data fusion dataset. Lastly, the fourth dataset is the combination of data fusion dataset and additional feature bands which comprise the RGB image, NIR, and NDVI image. The four datasets will be evaluated the performance on crop extraction following the experiment design.

\subsubsection{SegNet-Based Semantic Segmentation}

The aim of semantic segmentation is to generate human-interpretable labels for each pixel in a given image. Recently, $\mathrm{CNN}$ have been proved to be efficient and reliable models to achieve remarkable performance for image classification and object detection tasks [13], Moreover, it has been demonstrated that pre-trained $\mathrm{CNN}$ architectures can play an important role in terms of features extractors and allow high classification performance. [14]. The CNN model for crop detection classification presented in this section aims to provide a general architecture to be used with RS data from different sources and characteristics such as VRSS-2 images, Google imagery and fusion data. In this paper, we used SegNet architecture which is developed by Badrinarayanan, $\mathrm{V}$ et al. [15]. CNN used for semantic pixel-wise labeling commonly called semantic segmentation. Networks used for semantic segmentation took first an RGB image as input data which is a " $n$ " channel image, where " $n$ " is the number of labels involved. Each channel corresponded to a label, for example in this research was: banana, bare lands and pasture; then each pixel in a certain channel is given a numerical value depending on whether that pixel belongs to the labeling corresponding to that channel. The SegNet architecture is displayed in Figure 4 consists of layers called encoders and decoders. Each of the architecture's encoders applies a series of procedures such as: convolution, batch normalization and non-linearity, later it is applied max pooling on the result. Decoders are similar to the encoders, the 


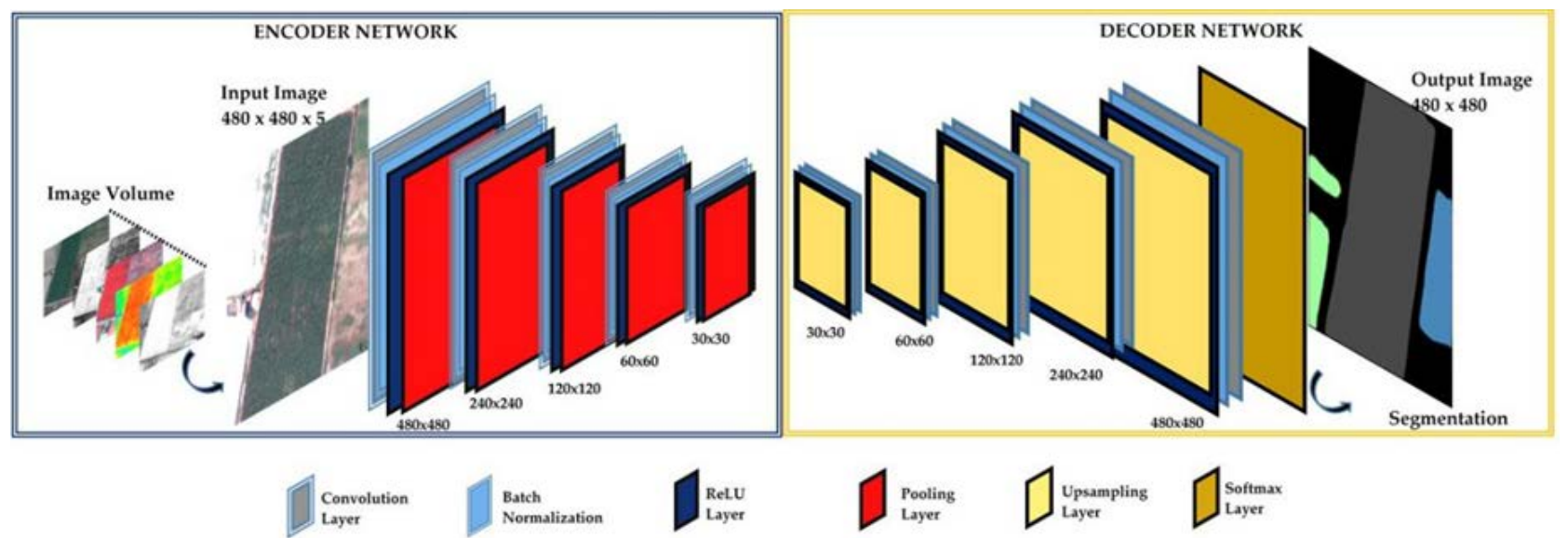

Figure 4. SegNet is composed of: the encoder (left) and the decoder (right). The input is an RGB image and the output is a segmentation image.

difference is that they do not have a non-linearity, and they up sample their input, using indices stored from the encoding stage. After the final decoder, the output is fed to a softmax classifier that gives the final prediction. SegNet is clearly structured and it is easy to understand the convolutional network of the model. In the model, dropout and batch normalization layers are introduced in the architecture, and dropout is applied at the fully connected layer, while batch normalization is used at every convolution. Furthermore, it was employed the ReLU function after all convolution layers. The max-pooling is applied with a stride of two in order to subsequently reduce the spatial dimension of the patch and focusing on the central pixel.

\subsection{Accuracy Assessment}

The semantic image segmentation challenge is evaluated using the mean Intersection over Union (mIoU) metric. The mIoU is a standard measure metric that represents the ratio of intersection to union between the prediction and ground truth. For evaluate the classification models was used overall accuracy (OA). In addition, the overall F1 score for all the classes in each data sets was computed.

\section{Results}

\subsection{Experiment: Crops Extraction Based on Google Imagery, VRSS-2 and Finally Data Fusion (RGB)}

The accuracy results of the proposed SegNet architecture it is displays in Figure 5. As it can be observed, the data fusion provides the most competitive results with an overall accuracy of $90.85 \%$, F1 score of $87.65 \%$ and mIoU of $83.26 \%$. It also achieves the high accuracies for every classes: banana $91.05 \%$, pasture 90.33 and bare lands 89.03. However, with only Google imagery and VRSS-2 data show a lower results which obtaining overall accuracies below $90 \%$. With regard to the VRSS-2 images results, it has a lower spatial resolution, the accuracy result is below to $86 \%$ since the pixels cover a larger area of land. It is difficult to 


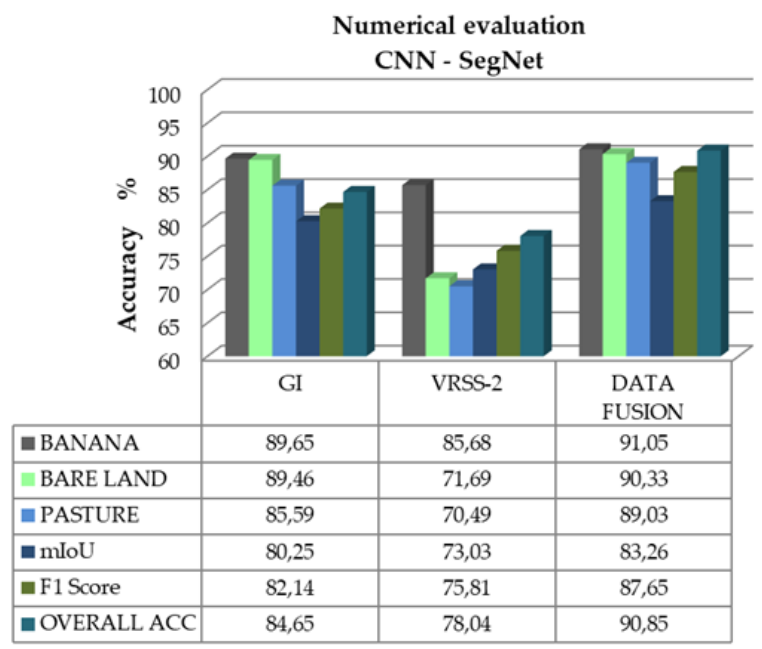

Figure 5. Cross validation overall accuracy of different data sets: only Google Imagery (GI), only VSRR-2 and data fusion only RGB images.

differentiate accurately the different types of crops which makes it more errors for SegNet to locate the common features, because of its structure containing a single convolutional network.

In the case of the pasture and bare land classes the model is able to distinguish some plantation with a low level of accuracy below to $75 \%$. The GI results show how large fields of different crops are properly mapped especially banana and bare land zones. Comparing the GI and VRSS-2 results, we see that GI yielded has 6\% increasing in overall accuracy and 7\% in F1 score. It demonstrates that crops extraction can boost classification performance for high-resolution imagery. However, the results present problems on pixels closer to the pasture area. Visual comparisons show that the segmentation results of SegNet architecture are more accurate in data fusion. Figure 6 shows different banana, bare land and pasture crops, but visually the samples grouped in left side they are a bit different with respect to the samples displayed in right side, because they have others patterns, color and shape, also shows diverse kind of pasture with mixed colors, managed through more intensive agricultural practices of seeding, even within the banana plantations there are irregular areas without crops, also the sunlit and shaded banana plant leaves and torn banana plant leaves and the bare lands with various color and some sample observations of different types of land conversions.

\subsection{Experiment 2: Crops Extraction Based on Data Fusion (RGB) and Additional Feature Bands}

The experiment results are shown in Figure 7. The data fusion with additional feature bands achieves an overall accuracy of $93.17 \%, \mathrm{~F} 1$ score of $90.16 \%$ and mIoU reports $85.94 \%$, whereas data fusion (RGB) achieves an overall accuracy of $90.85 \%, \mathrm{~F} 1$ score of $87.65 \%$ and mIoU reaches $83.26 \%$. It also achieves the high accuracies for every classes: banana $92.89 \%$, pasture $91.53 \%$ and bare lands 
(a)

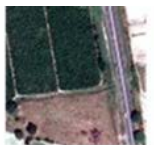

(b)

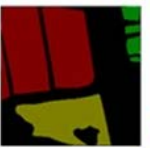

(c)

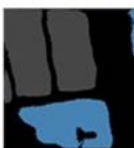

(d)

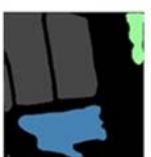

(e)

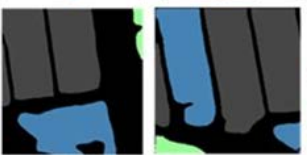

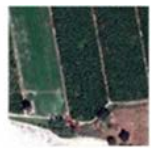
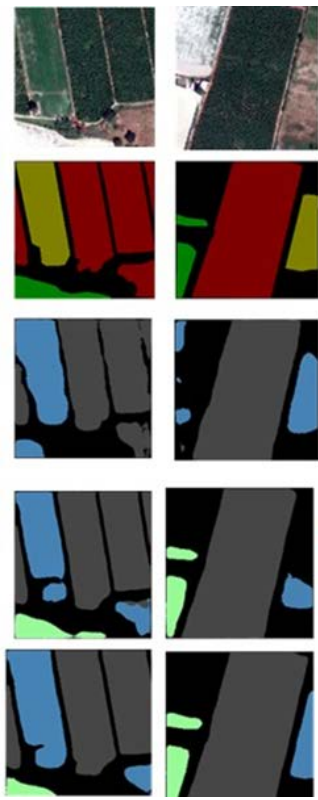
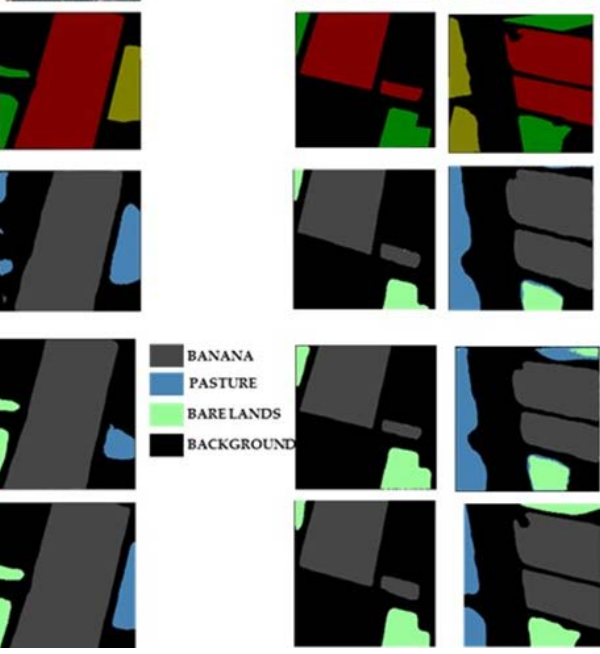
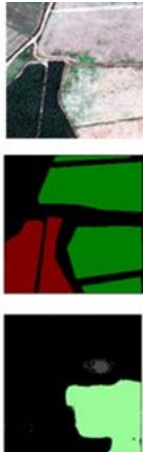

Figure 6. Visual segmentation results on testing set which consist of input ground true image (a), label data (b), crops extraction result from Google imagery (c), crops extraction result VRSS-2 RGB images (d) and crops extraction result Data fusion RGB images (e).

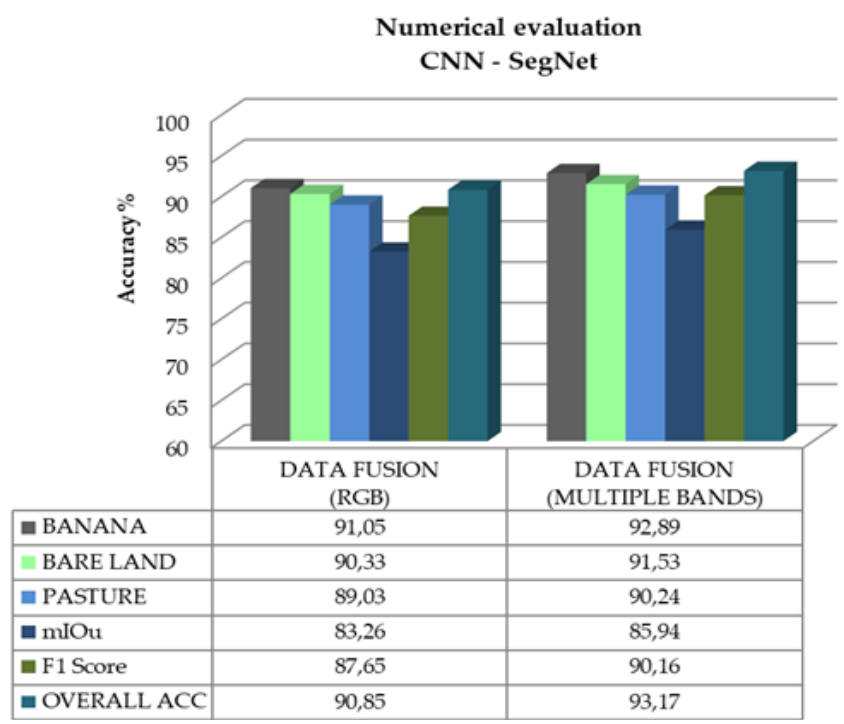

Figure 7. Cross validation overall accuracy between data fusion (RGB) and data fusion with multiple bands.

90.24\%. CNN-based crop detection with data fusion model achieved better detection results than data fusion with only RGB bands. On banana class achieved higher precision, $92.89 \%$ versus $91.05 \%$, bare lands, $91.53 \%$ versus $90.33 \%$, and pasture, $90.24 \%$ versus $89.03 \%$. More noticeable, on others data sets, data fusion with multiple bands achieved significantly better overall accuracy $93.17 \%$ versus 78.04\% (VRSS-2), and 84.65\% (GI). For both data fusion RGB and data fusion with additional feature bands, higher accuracies can be achieved compared to 
the use of each sensor individually.

The improvement of the results is due to the spectral fusion, spatial information and the vegetation indices. Meanwhile, when is add multiple bands the classification networks lead to higher overall accuracy, F1 score and mIoU for all three data sets. In addition, the proposed method achieves the highest accuracies on all classes. The results are also presented when the multiple bands are applied, which provides an average improvement of almost 3\% in accuracy. As it can be seen, the training data provided is limited with respect to public datasets. Nevertheless, the proposed network is able to produce smooth classification results for all cases, considering the three classes involved and the different characteristics of the images.

Comparing these results, we see that data fusion with multiple bands yielded a 2 percent increasing in overall accuracy. It demonstrates that VIs allowed the enhancing of the spectral information and increasing the separability of the interesting classes. Through the results, it is possible to verify that the datasets prepared with the data fusion with multiple bands are considerably better than the data from a single sensor such as Google imagery or VRSS-2. Visual comparisons show that the segmentation results of SegNet architecture are more accurate in data fusion. See Figure 8. Our CNN learnt and performed better on higher resolution images with multiple bands. When the image spatial resolution is high, and it added more additional bands extracted from the Vis. the accuracy show a better result. This also implies that, if $\mathrm{CNNs}$ are trained on high-resolution images with NDVI, GNDVI, ENDVI or other bands the performance will be better.

\section{Conclusion}

In this paper, we proposed a data fusion model based on SegNet architecture to

(a)

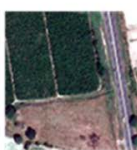

(b)

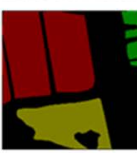

(c)

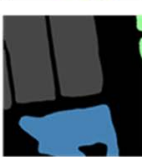

(d)

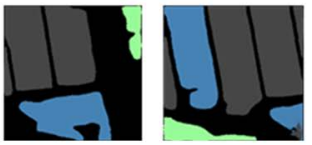

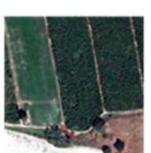
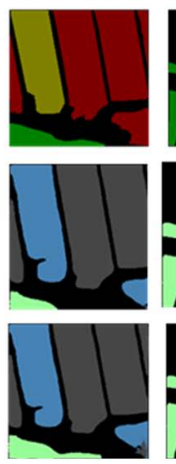
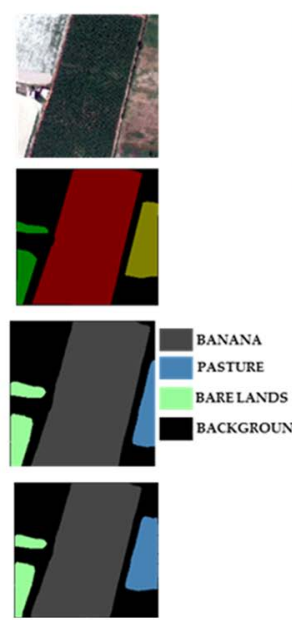
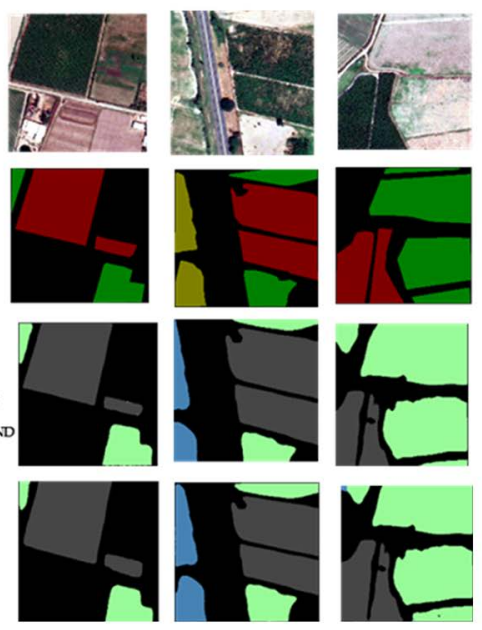

Figure 8. Visual segmentation results on testing set which consist of input ground true image (a), label data (b), crops extraction result from data fusion RGB (c) and crops extraction result Data fusion with multiple bands (d). 
perform crops detection with the remote sensing imagery of different sources. To build the training datasets, we selected two different area of the study area. The first one has been used to collect training data, and the second one was used for test data collection. Data fusion between VRSS-2 images and Google imagery was applied through the Gram Schmidt pan-sharpen method. Our study demonstrate that data fusion combined with DL is highly feasible to crop extraction from satellite images and GE imagery, as it has showed promising results for all the images have been studied, given that they are from different sources and distinct characteristics. It also proves that deep learning techniques can serve as invaluable tools for larger remote sensing data fusion frameworks. Moreover, data fusion model with the SegNet architecture allowed the full utilization of the strength of the convolution in the extraction of spatial semantics and made appropriate use of the rich information contained in the pixels of the remote sensing images, thus achieving a more accurate segmentation. The model presented in this paper provides a solution for the crop's extraction or the segmentation of the crop's plantation using VRSS-2 images and GI. The future work should include a deeper study on the data fusion level integrating lidar information with radar and hyper spectral images.

\section{Conflicts of Interest}

The authors declare no conflicts of interest regarding the publication of this paper.

\section{References}

[1] Lee, J., Seo, J. and Kang, S. (2018) Development of a Biophysical Rice Yield Model Using All-Weather Climate Data. Korean J. Remote Sens, 33, 721-732.

[2] Kim, Y., Park, N. and Lee, K.D. (2018) Self-Learning Based Land-Cover Classification Using Sequential Class Patterns from Past Land-Cover Maps. Remote Sens, 9, 921. https://doi.org/10.3390/rs9090921

[3] Senthilnath, J., Kandukuri, M., Dokania, A. and Ramesh, K.N. (2017) Application of UAV Imaging Platform for Vegetation Analysis Based on Spectral-Spatial Methods. Comput. Electron. Agric., 140, 8-24. https://doi.org/10.1016/j.compag.2017.05.027

[4] Hu, Q., et al. (2013) Exploring the Use of Google Earth Imagery and Object-Based Methods in Land Use/Cover Mapping. Remote Sens., 5, 6026-6042. https://doi.org/10.3390/rs5116026

[5] Hou, F., Lei, W., Li, H. and Xi, J. (2018) FMRSS Net: Fast Matrix Representation-Based Spectral-Spatial Feature Learning Convolutional Neural Network for Hyperspectral Image Classification. Math. Probl. Eng., 2018, Article ID: 9218092. https://doi.org/10.1155/2018/9218092

[6] Pound, M., et al. (2016) Deep Machine Learning Provides State-of-the-Art Performance in Image-Based Plant Phenotyping. bioRxiv, 053033.

[7] Di Cicco, M., Potena, C., Grisetti, G. and Pretto, A. (2016) Automatic Model Based Dataset Generation for Fast and Accurate Crop and Weeds Detection. 2017 IEEE/RSJ International Conference on Intelligent Robots and Systems (IROS), Vancouver, BC, 24-28 Sept. 2017. https://doi.org/10.1109/IROS.2017.8206408

[8] Johannes, A., et al. (2017) Automatic Plant Disease Diagnosis Using Mobile Capture 
Devices, Applied on a Wheat Use Case. Comput. Electron. Agric, 138, 200-209. https://doi.org/10.1016/j.compag.2017.04.013

[9] Liang-Chieh, C., Papandreou, G., Kokkinos, I., Murphy, K. and Yuille, A. (2015) Semantic Image Segmentation with Deep Convolutional Nets and Fully Connected CRFs. Proc. Int. Conf. Learn., Representations.

[10] Yu, L. and Gong, P. (2011) Google Earth as a Virtual Globe Tool for Earth Science Applications at the Global Scale: Progress and Perspectives. Int. J. Remote Sens, 33, 3966-3986. https://doi.org/10.1080/01431161.2011.636081

[11] Dragut, L., Tiede, D. and Levick, S. (2010) ESP: A Tool to Estimate Scale Parameter for Multiresolution Image Segmentation of Remotely Sensed Data. Int. J. Geogr. Inf. Sci, 24, 859-871. https://doi.org/10.1080/13658810903174803

[12] Li, H., Ding, W., Cao, X. and Liu, C. (2017) Image Registration and Fusion of Visible and Infrared Integrated Camera for Medium-Altitude Unmanned Aerial Vehicle Remote Sensing. Remote Sens, 9, 441. https://doi.org/10.3390/rs9050441

[13] Boonpook, W., et al. (2018) A Deep Learning Approach on Building Detection from Unmanned Aerial Vehicle-Based Images in Riverbank Monitoring. Sensors, 18, 3921. https://doi.org/10.3390/s18113921

[14] Cascio, D., Taormina, V. and Raso, G. (2019) Deep Convolutional Neural Network for HEp-2 Fluorescence Intensity Classification. Appl. Sci, 9, 408. https://doi.org/10.3390/app9030408

[15] Badrinarayanan, V., Kendall, A. and Cipolla, R. (2017) SegNet: A Deep Convolutional Encoder-Decoder Architecture for Image Segmentation. IEEE Trans. Pattern Anal. Mach. Intell, 39, 2481-2495. https://doi.org/10.1109/TPAMI.2016.2644615 